\title{
Risk Prediction for Locoregional Recurrence in Epidermal Growth Factor Receptor-Mutant Stage III-pN2 Lung Adenocarcinoma after Complete Resection: A Multi-center Retrospective Study
}

Qi-Wen $\mathrm{Li}^{1,2^{*}}$, Bo Qiu ${ }^{1,2^{*}}$, Wen-Hua Liang ${ }^{3^{*}}$, Jun-Ye Wang ${ }^{2,4^{*}}$, Wan-Ming Hu ${ }^{2,5}$, Tian Zhang ${ }^{6}$, Shuang-Bing $\mathrm{Xu}^{7}$, José López ${ }^{8}$, Nai-Bin Chen ${ }^{1,2}$, Min-Zhang Guo ${ }^{3}$, Yi Zhao ${ }^{3}$, Ling-Juan Chen ${ }^{7}$, Song-Ran Liu',5, Jing-Ping Yun 2,5, Jin-Yu Guo 1,2, Si-Yu Wang2,4, Xin Wang2,4, Li Zhang2,9, Dong-Sheng Yue ${ }^{10,}$ Zhong-Xing Liao11, Steven H. Lin ${ }^{11}$, Hao Long ${ }^{2,4}$, Qing-Song Pang ${ }^{\natural}$, Hui Liu ${ }^{1,2} \bowtie$

1. Department of Radiation Oncology, State Key Laboratory of Oncology in South China, Collaborative Innovation Center for Cancer Medicine, Sun Yat-sen University Cancer Center, Guangzhou, China

2. Lung Cancer Research Center, Sun Yat-sen University, Guangzhou, China

3. Department of Thoracic Surgery and Oncology, The First Affiliated Hospital of Guangzhou Medical University/State Key Laboratory of Respiratory Disease/National Clinical Research Center of Respiratory Disease, Guangzhou, China

4. Department of Thoracic Surgery, State Key Laboratory of Oncology in South China, Collaborative Innovation Center for Cancer Medicine, Sun Yat-sen University Cancer Center, Guangzhou, China

5. Department of Pathology, State Key Laboratory of Oncology in South China, Collaborative Innovation Center for Cancer Medicine, Sun Yat-sen University Cancer Center, Guangzhou, China

6. Department of Radiation Oncology, Tianjin Medical University Cancer Institute and Hospital, Tianjin, China

7. Union Hospital Cancer Center, Tongii Medical College, Huazhong University of Science and Technology, Wuhan, China

8. Group of Technological Innovation, Radiation Oncology, University Hospital Virgen del Rocio, Sevilla, Spain

9. Department of Medical Oncology, State Key Laboratory of Oncology in South China, Collaborative Innovation Center for Cancer Medicine, Sun Yat-sen University Cancer Center, Guangzhou, China

10. Department of Lung Cancer, Lung Cancer Center, Tianjin Medical University Cancer Institute and Hospital, Tianjin, China

11. Department of Radiation Oncology, M.D. Anderson Cancer Center, Houston, Texas, USA

${ }^{*} \mathrm{Qi}-\mathrm{Wen} \mathrm{Li}$, Bo Qiu, Wen-Hua Liang and Jun-Ye Wang contributed equally to this work.

$\square$ Corresponding authors: Hui Liu, Department of Radiation Oncology, Sun Yat-sen University Cancer Center, No.651 Dongfeng Road East, Guangzhou, 510060, China. E-mail: liuhuisysucc@sina.com. Tel: 8620-87343031. Fax number: 8620-87343492. Qing-Song Pang, Department of Radiation Oncology, Tianjin Medical University Cancer Institute and Hospital, Huan-Hu-Xi Road, Ti-Yuan-Bei, He Xi District, Tianjin 300060, China. E-mail: pangqingsong@tjmuch.com. Tel: 8622-23340123-1121. Fax number: 8622-23359904. Hao Long, Department of Thoracic Surgery, Sun Yat-sen University Cancer Center, No.651 Dongfeng Road East, Guangzhou, 510060, China. E-mail: longhao@sysucc.org.cn. Tel: 8620-87343261. Fax number: 8620-87343492

(c) The author(s). This is an open access article distributed under the terms of the Creative Commons Attribution License (https://creativecommons.org/licenses/by/4.0/). See http://ivyspring.com/terms for full terms and conditions.

Received: 2020.04.17; Accepted: 2020.08.12; Published: 2020.08.25

\begin{abstract}
Background: This study aimed to develop a predictive model based on the risk of locoregional recurrence (LRR) in epidermal growth factor receptor (EGFR)-mutant stage III-pN2 lung adenocarcinoma after complete resection.

Methods: A total of 11,020 patients with lung surgery were screened to determine completely resected EGFR-mutant stage III-pN2 lung adenocarcinoma. Patients were excluded if they received preoperative therapy or postoperative radiation therapy (PORT). The time from surgery to LRR was recorded. Clinicopathological variables with statistical significance predicting LRR in the multivariate Cox regression were incorporated into the competing risk nomogram. Patients were then sub-grouped based on different recurrence risk as a result of the nomogram.

Results: Two hundred and eighty-eight patients were enrolled, including 191 (66.3\%) with unforeseen N2 (IIIAI-2), 75 (26.0\%) with minimal/single station N2 (IIIA3), and 22 (7.6\%) with bulky and/or multilevel N2 (IIIA4). The 2-year overall cumulative incidence of LRR was $27.2 \%$ (confidence interval [Cl], $16.3 \%-38.0 \%$ ). IIIA4 disease (hazard ratio, $2.65 ; \mathrm{Cl}, 1.15-6.07 ; P=0.022$ ) and extranodal extension (hazard ratio, 3.33; $\mathrm{Cl}, 1.76-6.30 ; P<0.001$ ) were independent risk factors for $L R R$ and were incorporated into the
\end{abstract}


nomogram. Based on the nomogram, patients who did not have any risk factor (low-risk) had a significantly lower predicted 2-year incidence of LRR than those with any of the risk factors (high-risk; $4.6 \%$ vs $21.9 \%, P<0.001)$.

Conclusions: Pre-treatment bulky/multilevel N2 and pathological extranodal extension are risk factors for locoregional recurrence in EGFR-mutant stage III-pN2 lung adenocarcinoma. Intensive adjuvant therapies and active follow-up should be considered in patients with any of the risk factors.

Key words: Locoregional recurrence; postoperative radiotherapy; lymph node metastasis; extranodal extension; Robinson classification

\section{Introduction}

Locoregional recurrence (LRR) has been reported in over $20 \%-30 \%$ of patients with stage IIIA lung adenocarcinoma.[1-2] Postoperative radiation therapy (PORT) has a theoretical potential to improve locoregional control and other long-term outcomes in stage III-pN2 patients, which is also supported by results from large-scale retrospective studies.[3-5] However, stage III-pN2 patients consist of heterogenous subgroups with significantly different LRR risk, thus only $29 \%-64 \%$ of $\mathrm{pN} 2$ patients were referred to PORT in clinical practice [3-5]. The commonly recognized risk factors include the status of lymph node involvement and certain histological characteristics,[9] but most of them are experience-based rather than evidence-based. A precise identification of the high-risk patients is important for the recommendation of PORT administration.

Suggested by our previous study, epidermal growth factor receptor (EFGR) mutation was associated with a lower risk of LRR in completely resected stage III-pN2 lung adenocarcinoma. Among these patients, only $18.6 \%$ of all noted recurrences occurred in the locoregional site, which was significantly lower than those of EGFR wild-type counterparts (36.7\%).[10] Besides, recent reports revealed even better disease control with the administration of adjuvant tyrosine kinase inhibitor (TKI) compared with adjuvant chemotherapy.[11-12] Based on the evidence, we made a hypothesis that PORT might not be beneficial in most patients with EGFR-mutant stage III-pN2 lung adenocarcinoma, except for those with extra risk factors for LRR.

We performed the retrospective study to investigate the risk factors of LRR in EGFR-mutant stage III-pN2 lung adenocarcinoma after complete surgical resection. A nomogram was developed to subgroup patients with high and low LRR risk. The study will provide important evidence on further investigation on PORT candidates. Omission of PORT might be recommended for patients with extremely low risk of LRR, and the potential of PORT should be only examined in those with higher chance of LRR in future studies.

\section{Patients and Methods}

\section{Ethics, consent and permissions}

The study was conducted according to the ethical standards of the Helsinki Declaration. It was reviewed and approved by the Ethics Committee of Sun Yat-sen University Cancer Center (YB2017-047). Since it was a retrospective and anonymous study, a waiver of authorization was required and granted.

\section{Study population}

Consecutive patients who had lung surgery and were sent for the EGFR mutation test during the time-frame spanning from September 2001 to December 2016 at either of Sun Yat-sen University Cancer Center, Tianjin Medical University Cancer Institute and Hospital, Union Hospital Cancer Center and The First Affiliated Hospital of Guangzhou Medical University were retrospectively screened. The following criteria were met for study inclusion: 1) histologically confirmed lung adenocarcinoma with sensitizing EGFR mutation (exon 19 or 21); 2) regarded as resectable according to pre-operative work-ups, including chest and upper abdominal computed tomography (CT) scans, bone scan, brain magnetic resonance imaging (MRI) and bronchoscopy, and in some cases, positron emission tomography (PET)/CT scan, endobronchial ultrasound-guided transbronchial needle aspiration (EBUS-TBNA) or mediastinoscopy; 3) resected completely; and 4) pathologically diagnosed with N2 disease. Patients were excluded if they received induction therapy or PORT, or had a postoperative follow-up time of less than six months. The staging was based on AJCC/UICC $8^{\text {th }}$ staging criteria.[13]

\section{N sub-staging}

$\mathrm{N}$ sub-staging was collected and examined as a potential risk factor of LRR. Pre-treatment work-ups and pathology reports were carefully reviewed to confirm the status of N2 nodes. They were further sub-staged as unforeseen N2 (IIIA1-2), minimal $\mathrm{N} 2$ / single station at staging (IIIA3), and bulky and/or 
multilevel N2 at staging (IIIA4), according to the Robinson Classification.[14] IIIA1 was not separated from IIIA2 because intraoperative mediastinal lymph node pathological staging was not routinely performed.

\section{EGFR genotyping}

Paraffin-embedded, formalin-fixed tissues obtained from surgical samples were prepared for the extraction of genomic DNA. EGFR mutation was tested via the amplification-refractory mutation system (ARMS) or direct sequencing depending on the technique used in each center.

\section{Pathological examination}

An experienced pathologist, one for each center, reviewed the slides with hematoxylin-eosin staining, immunohistochemical staining and elastic staining. Complete resection was defined as free resection margins proven microscopically. The numbers of examined nodes were counted to reflect the quality of both lymphadenectomy and pathological examination. A cut-off of 16 examined nodes was used for further analysis because our previous work suggested it as a prognostic factor of overall survival (OS).[15] Extranodal extension was defined as the invasion of malignant cells into perinodal adipose tissue through the nodal capsule. Tumor extension beyond the elastic layer, into the lymph and vascular system, or into the space surrounding a nerve was considered as visceral pleural invasion, lymphovascular invasion or perineural invasion, respectively. Skip lymph node metastasis was defined as N2 involvement without positive N1 node.[16]

\section{Surgery}

Before surgery, all patients with multilevel or/and bulky N2 but refused neoadjuvant chemotherapy were discussed by a multidisciplinary team (MDT). The patients went straight to surgery unless that the tumor was regarded primarily resectable. The surgical procedure was decided according to the size and location of disease, patients' pulmonary function, cardiac function, and other comorbidities. Ipsilateral station 1 nodes were routinely dissected. Ipsilateral mediastinal lymph node dissection was performed, including the dissection of stations $2 \mathrm{R}, 4 \mathrm{R}$, and 7-9 for right lung cancer and 4L, 5, 6, and 7-9 for left lung cancer.

\section{Adjuvant therapies}

The adjuvant administration of chemotherapy (at least four cycles) and TKIs (at least two months) were reviewed and documented. Adjuvant chemotherapy was routinely recommended for all patients, in the light of the current guidelines.[6-7]
Either of the following two-drug regimens was administered with recommended dose: pemetrexed+ cisplatin/carboplatin, paclitaxel +carboplatin, docetaxel+cisplatin/nedaplatin, vinorelbine detartrate+cisplatin, or gemcitabine+cisplatin/nedaplatin. Some patients treated between 2012 and 2015 in Tianjin Medical University Cancer Institute and Hospital were enrolled in a phase 2 clinical trial (NCT01683174), where the participants were randomized for adjuvant erlotinib or vinorelbine plus cisplatin. For those refused or showed intolerance to adjuvant chemotherapy, adjuvant TKI was discussed by patients and physicians as a substitute. Either gefitinib, erlotinib, afatinib or icotinib was administered. In some cases, after standard adjuvant chemotherapy, a maintenance use of TKIs was decided if an agreement was reached by patients and physicians, after weighing pros and cons.

\section{Follow-up}

Chest and upper abdominal CT, and brain MRI were performed 1-2 months after the end of treatment, every 3-6 months in the first two years, and every 6-12 months after that.[6] Bone scan, PET/CT and biopsy were performed if necessary. Any relapse within the ipsilateral hemithorax (except for multiple recurrent lesions in the ipsilateral lung) or regional lymph nodes was regarded as a locoregional recurrence; relapse elsewhere was considered distant metastasis.[17] Both ultimate local and distant disease progression were documented.

\section{Statistical methods}

The time from surgery to first locoregional relapse, first distant metastasis, death or last follow-up were recorded. The cumulative incidence function was used to calculate the probability of LRR and distant metastasis (DM), where death was considered as a competing event.[18] Disease-free survival (DFS) was defined as the time from surgery to the first recorded treatment failure or death. OS was defined as the time from surgery to death from any cause. DFS and OS were assessed by the Kaplan-Meier method. The associations between clinicopathological variables and the incidence of LRR were assessed by employing competing risk regression analysis.[18] Factors with $P<0.10$ in univariable analyses were incorporated into the proportional subdistribution hazard model and evaluated in multivariate analyses. P-values $<0.05$ (two-sided) were regarded as statistically significant. Missing data were not included in the statistical analysis. Statistical tests were conducted using SPSS 22.0 and R 3.0.2. 


\section{Nomogram}

Variables achieving statistical significance in the multivariable analysis were used to formulate a competing risk nomogram by $R$ 3.0.2.[19] The nomogram was built to evaluate the chance of LRR and to select high-risk patients for intensive adjuvant therapies in future. A high recurrence risk was defined as a nomogram-predicted 2-year cumulative incidence of LRR exceeding 10\%. PORT might be spared in those with a low recurrence risk, while intensive adjuvant therapies should be considered in high-risk patients in future. Internal validations were performed. A concordance index (c-index) value was calculated to measure discrimination performance, and a calibration curve was obtained by plotting the observed incidence against the nomogram-predicted probability via a bootstrap method with 1000 resamples.

\section{Results}

A total of 11,020 patients were screened. Among these, 288 consecutive cases met the study criteria and were included in the analysis (Fig. 1). Table 1 details the clinicopathological characteristics and treatment-related parameters. There were 191 (66.3\%) patients with IIIA1-2, 75 (26.0\%) with IIIA3, and 22 (7.6\%) with IIIA4. The 22 patients with IIIA4 did not receive adjuvant radiotherapy, due to contraindications $(n=14)$, patient's refusal $(n=3)$, economic reasons $(n=3)$ or MDT recommendations $(\mathrm{n}=2)$. There were $152(52.8 \%)$ patients diagnosed with exon 19 deletion, and $136(47.2 \%)$ with exon 21 mutation, including 134 with L858R mutation and 2 with L861Q mutation. One hundred and ninety-four $(67.4 \%)$ patients received adjuvant chemotherapy, while $67(23.3 \%)$ received adjuvant TKIs. Of those had adjuvant TKIs, 20 were included in a clinical trial (NCT01683174), and 26 refused or were intolerant to adjuvant chemotherapy; the other 21 patients had adjuvant chemotherapy followed by adjuvant TKIs based on an individual decision made by the patients and physicians. The detailed regimens of adjuvant chemotherapy and TKIs are presented in Table S1.

\section{Patterns of recurrence}

The median follow-up time was 28 (range: 6-133) months. Among the 288 patients, 46.5\% (134/288) experienced relapse. Ultimately, 5.2\% (15/288) had local recurrence only, $28.1 \%(81 / 288)$ had distant metastases only, $10.1 \%(29 / 288)$ had both, and 3.1\% $(9 / 288)$ had recurrence at unknown sites. The 2-year incidences of LRR and DM were $27.2 \%$ (CI, $16.3 \%-38.0 \%)$ and $58.2 \% \quad(\mathrm{CI}, 37.0 \%-79.3 \%)$, respectively. The sites of locoregional and distant relapses were detailed in Table S2.
After the first failure, 9 patients $(6.7 \%, 9 / 134)$ had traditional Chinese medicine or palliative therapy because of economic reasons or personal decisions. The other 125 patients received salvage treatments including: TKI alone $(\mathrm{n}=72)$, chemotherapy alone $(n=16), \quad$ TKI+surgery $\quad(n=16), \quad$ TKI+radiotherapy $(n=15)$, chemotherapy+radiotherapy $(n=4)$, and surgery alone $(n=2)$.

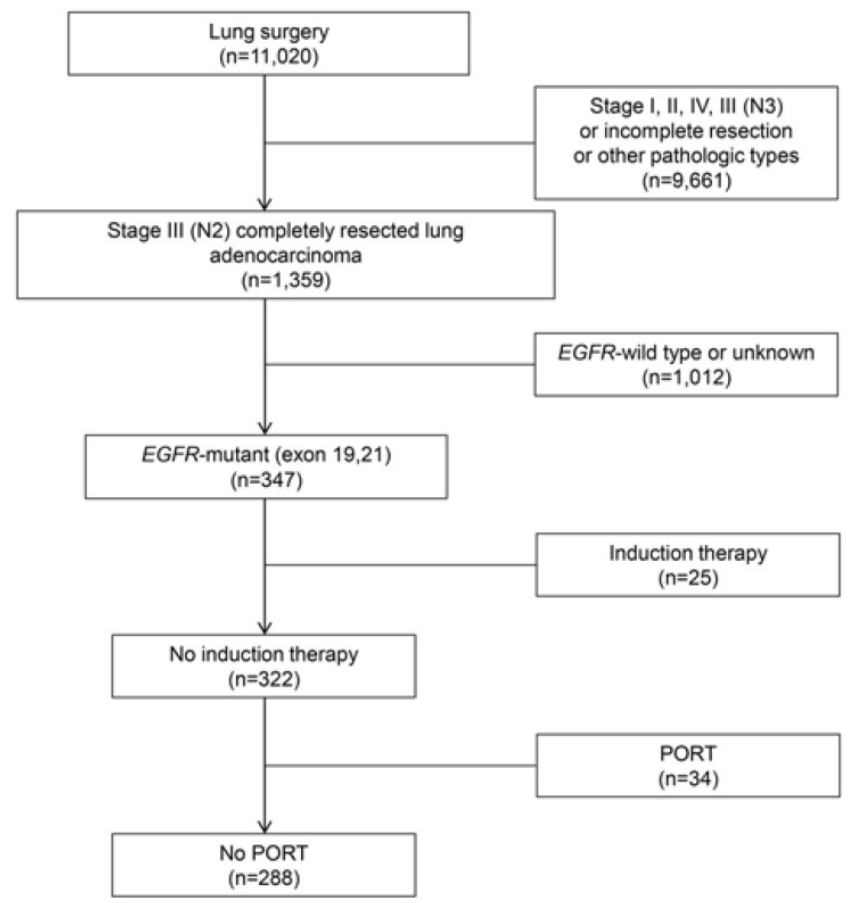

Fig. 1. Flowchart for patient enrollment. EGFR: epidermal growth factor receptor; PORT: postoperative radiation therapy.

\section{Risk factors and predictive nomogram for LRR}

The N2 substage was associated with local recurrence, reflected by a 2-year incidence of LRR of $25.4 \%$ in IIIA4 patients and $9.8 \%$ in IIIA1-3 patients $(P=0.033$, Fig. 2A). Another risk factor for 2-year LRR rate was extranodal extension $(21.2 \%$ vs. $6.2 \%$, $P<0.001$, Fig. 2B). No statistically significant difference in LRR rates was observed between patients with or without adjuvant TKI treatment (2-year incidence of LRR, $7.9 \%$ vs $12.4 \%, P=0.22$ ). In multivariate analysis, IIIA4 disease (hazard ratio [HR], 2.65; confidence interval [CI], 1.15-6.07; $P=0.022$ ) and extranodal extension (HR, 3.33; CI, 1.76-6.30; $P<0.001)$ were demonstrated as independent risk factors (Table 2).

A nomogram predicting the 1-, 2- and 3-year cumulative incidences of LRR was created incorporating N2 status and pathological extranodal extension (Fig. 3A). The results of internal validation showed that the nomogram had a c-index of $0.715(\mathrm{CI}$, 0.695-0.735) and was well calibrated for patients with low LRR risk (Fig. 3B). With a cut-off value of LRR 
risk of $10 \%$, the patients were divided into the low-risk group (IIIA1-3 and no extranodal extension, $62.5 \%, 155 / 248$ ) and the high-risk group (IIIA4 disease or/and extranodal extension, 37.5\%, 93/248). The 2-year incidence of LRR was significantly different between the groups (low- vs high-risk groups, $4.6 \%$ vs $21.9 \%, P<0.001$, Fig. $2 \mathrm{C}$ ).

\section{Survivals}

At the latest follow-up, 67 deaths were recorded among the whole cohort. The 2-year DFS and OS were $58.0 \%$ (CI, 51.7\%-64.3\%) and 89.7\% (CI, 85.8\%-93.6\%), respectively. The estimated median DFS and OS were 28.0 (CI, 22.4-33.6) and 70.0 (CI, 61.0-79.0) months, respectively.

Table 1. Patient characteristics

\begin{tabular}{|c|c|}
\hline Characteristics & All patients \\
\hline & $\mathrm{n}=288(\%)$ \\
\hline \multicolumn{2}{|l|}{ Age } \\
\hline$\geq 60$ & $126(43.7)$ \\
\hline$<60$ & $162(56.3)$ \\
\hline \multicolumn{2}{|l|}{ Sex } \\
\hline Male & $108(37.5)$ \\
\hline Female & $180(62.5)$ \\
\hline \multicolumn{2}{|l|}{ KPS } \\
\hline $90-100$ & $280(97.2)$ \\
\hline 80 & $8(2.8)$ \\
\hline \multicolumn{2}{|c|}{ Pre-treatment $\mathrm{T}$ stage } \\
\hline $\mathrm{T} 1-2$ & $270(93.8)$ \\
\hline T3-4 & $18(6.2)$ \\
\hline \multicolumn{2}{|c|}{ Pre-treatment N stage } \\
\hline No & $88(30.5)$ \\
\hline N1 & $103(35.8)$ \\
\hline N2 & $97(33.7)$ \\
\hline \multicolumn{2}{|l|}{ Surgery } \\
\hline Lobectomy & $279(96.9)$ \\
\hline Pneumonectomy & $9(3.1)$ \\
\hline \multicolumn{2}{|l|}{ EGFR mutation } \\
\hline Exon 19 & $152(52.8)$ \\
\hline Exon 21 & $136(47.2)$ \\
\hline \multicolumn{2}{|l|}{ Smoking } \\
\hline Yes & $72(25.0)$ \\
\hline No & $216(75.0)$ \\
\hline \multicolumn{2}{|l|}{ N2 classification } \\
\hline IIIA1-3 & $266(92.4)$ \\
\hline IIIA4 & $22(7.6)$ \\
\hline \multicolumn{2}{|c|}{ Number of examined nodes } \\
\hline$<16$ & $91(32.4)$ \\
\hline$\geq 16$ & $190(67.6)$ \\
\hline Missing data & 7 \\
\hline \multicolumn{2}{|c|}{ Visceral pleural invasion } \\
\hline Yes & $94(33.7)$ \\
\hline No & $185(66.3)$ \\
\hline Missing data & 9 \\
\hline \multicolumn{2}{|c|}{ Lymphovascular invasion } \\
\hline Yes & $99(39.3)$ \\
\hline No & $153(60.7)$ \\
\hline Missing data & 36 \\
\hline \multicolumn{2}{|c|}{ Perineural invasion } \\
\hline Yes & $12(5.3)$ \\
\hline No & $215(94.7)$ \\
\hline Missing data & 61 \\
\hline \multicolumn{2}{|c|}{ Extranodal extension } \\
\hline Yes & $78(31.5)$ \\
\hline No & $170(68.5)$ \\
\hline Missing data & 40 \\
\hline
\end{tabular}

\begin{tabular}{ll}
\hline Characteristics & All patients \\
\hline Skip metastasis of lymph node & \\
Yes & $80(29.0)$ \\
No & $196(71.0)$ \\
Missing data & 12 \\
pT stage & \\
T1-2 & $260(90.3)$ \\
T3-4 & $28(9.7)$ \\
Adjuvant TKIs & \\
Yes & $67(23.3)$ \\
No & $221(76.7)$ \\
Adjuvant chemotherapy & $194(67.4)$ \\
Yes & $94(32.6)$ \\
No & PORT: postoperative radiation therapy; KPS: Karnofsky performance score; EGFR: \\
epidermat growth factor receptor; IIIA1-2: unforeseen N2; IIIA3: minimal/single \\
station N2; IIIA4: bulky and/or multilevel N2; TKI: tyrosine kinase inhibitor. \\
Missing data was presented but not included in analysis.
\end{tabular}

Table 2. Prognostic factors for LRR

\begin{tabular}{|c|c|c|c|}
\hline & $\begin{array}{l}\text { Univariable } \\
\text { analysis }\end{array}$ & $\begin{array}{l}\text { Multivariable } \\
\text { analysis }\end{array}$ & \\
\hline Variable & $P$ & HR $(95 \% \mathrm{CI})$ & $P$ \\
\hline Age $(\geq 60 v s<60)$ & 0.64 & & \\
\hline Sex (male $v$ s female) & 0.86 & & \\
\hline Pre-treatment $\mathrm{T}$ stage (T1-2 vs T3-4) & 0.78 & & \\
\hline Pre-treatment $\mathrm{N}$ stage ( $\mathrm{N} 0$ vs $\mathrm{N} 1$ vs $\mathrm{N} 2)$ & 0.22 & & \\
\hline $\begin{array}{l}\text { Surgery (Lobectomy vs } \\
\text { Pneumonectomy) }\end{array}$ & 0.55 & & \\
\hline EGFR mutation (exon 19 vs 21) & 0.064 & $\begin{array}{l}1.72 \\
(0.85-3.57)\end{array}$ & 0.13 \\
\hline Smoking (yes vs no) & 0.92 & & \\
\hline N2 classification (IIIA4 vs IIIA1-3) & 0.033 & $\begin{array}{l}2.65 \\
(1.15-6.07)\end{array}$ & 0.022 \\
\hline $\begin{array}{l}\text { Number of examined nodes ( }<16 \text { vs } \\
\geq 16 \text { ) }\end{array}$ & 0.68 & & \\
\hline Visceral pleural invasion (yes $v s$ no) & 0.31 & & \\
\hline Lymphovascular invasion (yes vs no) & 0.66 & & \\
\hline Perineural invasion (yes $v s$ no) & 0.95 & & \\
\hline Extranodal extension (yes $v s$ no) & $<0.001$ & $\begin{array}{l}3.33 \\
(1.76-6.30)\end{array}$ & $<0.001$ \\
\hline $\begin{array}{l}\text { Skip metastasis of lymph node (yes } v s \\
\text { no) }\end{array}$ & 0.27 & & \\
\hline pT stage (T1-2 vs T3-4) & 0.94 & & \\
\hline Adjuvant TKIs (Yes vs No) & 0.22 & & \\
\hline Adjuvant chemotherapy (Yes $v s$ No) & 0.29 & & \\
\hline
\end{tabular}

\section{Discussion}

Our study investigated the patterns of recurrence and risk factors for LRR in EGFR-mutant stage III-pN2 lung adenocarcinoma after complete resection. Consecutive patients from four high-volume medical centers in China were combined. To the best of our knowledge, this was the first large retrospective study focusing on locoregional recurrence status in this specific subgroup.

In the current study, locoregional recurrence accounted for $15.3 \%$, which was much less than that of distant metastasis (38.2\%). The results were in good agreement with our previous study, in which local recurrence was $10.8 \%$ and $22 \%$ in EGFR-mutant and wild-type lung adenocarcinoma, respectively.[10] 

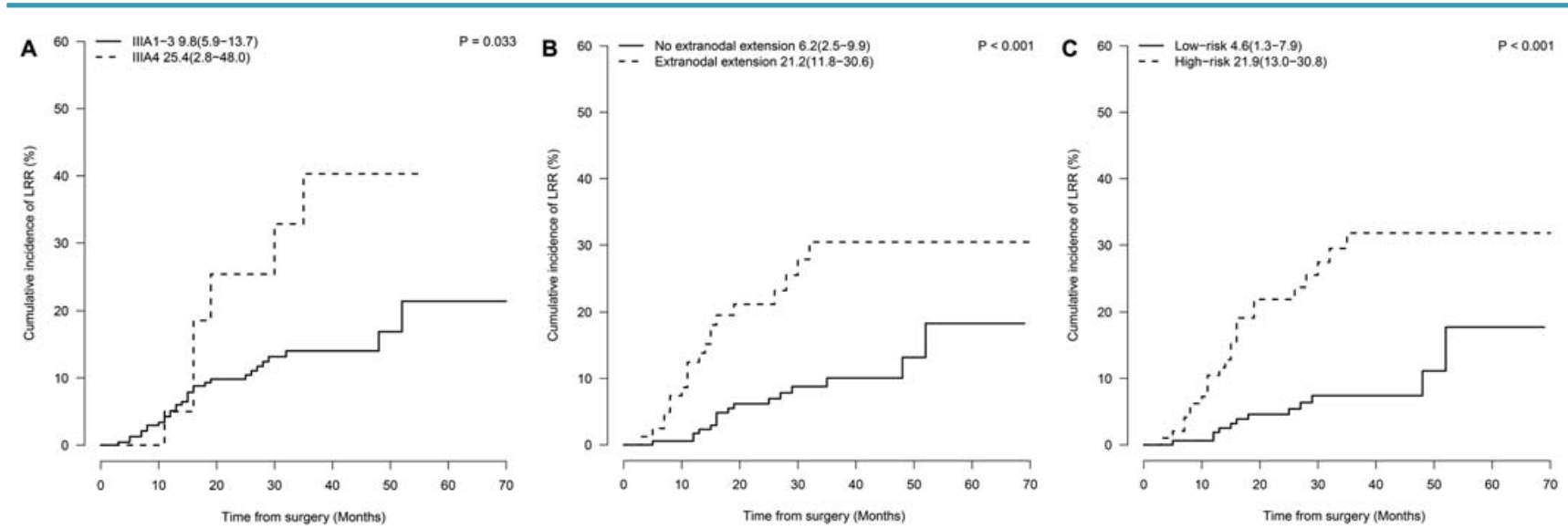

Fig. 2. Prognostic factors of locoregional recurrence. Cumulative incidences of LRR by (A) N2 classification (2-year cumulative incidence of LRR, IIIA1-3 vs IIIA4: $9.8 \%$ vs $25.4 \%, P=0.033$ ); (B) extranodal extension (2-year cumulative incidence of $L R R$, Yes vs No: $21.2 \%$ vs $6.2 \%, P<0.001$ ); (C) risk-group (2-year cumulative incidence of $L R R$, low-risk vs high-risk group: $4.6 \%$ vs $21.9 \%, P<0.001)$. LRR: locoregional recurrence.

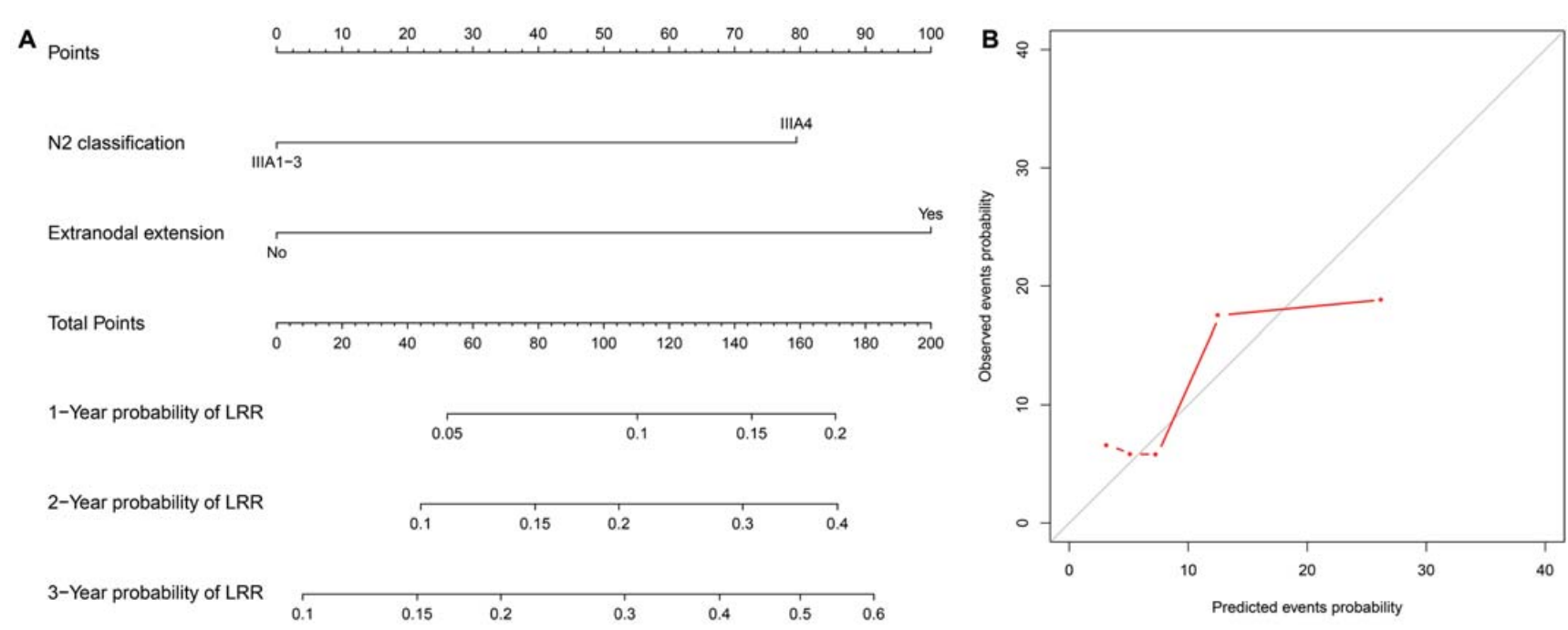

Fig. 3. LRR nomogram and calibration curves. (A) The nomogram is created to estimate the 1-, 2- and 3-year cumulative incidence of LRR. The value of one individual patient is located at each variable axis. A straight line drawn upward determines the points received for each variable value. The sum of points is located on the total point axis, and then a straight line is drawn downward to the cumulative incidence of LRR axes to determine the 1-, 2-, or 3-year cumulative incidence of LRR. (B) The calibration curves predict the cumulative incidence of LRR at 1, 2 and 3 years. LRR: locoregional recurrence.

Comparably, Mak et al.[20] retrospectively examined locally advanced lung adenocarcinoma patients treated by chest radiotherapy. The rates of local recurrence and distant metastasis were reported $24 \%$ and $79 \%$ of EGFR-mutant patients, and in $46 \%$ and $66 \%$ of wild-type patients.

The presence of regional lymph nodes at initial presentation provides important information on the severity and behavior of stage III-pN2 lung adenocarcinoma. Robinson et al. [14] proposed four degrees of pre-treatment N2, demonstrating increasing aggressiveness of mediastinal nodes and difficulty in the radical resection. Distinct from wild-type patients, who had a higher chance (about $50 \%)$ to be diagnosed with IIIA4, the majority (66.3\%) of EGFR-mutant patients had more unforeseen N2 (IIIA1-2).[21] Unforeseen N2 reflects minimal mediastinal invasion and favorable survival.[22,23] In this subgroup, the addition of adjuvant radiotherapy to chemotherapy did not provide extra benefit compared with chemotherapy alone.[24] Minimal N2/single station was regarded as potentially resected cases proposed by the European Society for Medical Oncology (ESMO) Clinical Practice Guidelines.[7] However, our data showed similar local control rates in the IIIA3 and IIIA1-2 subgroups, regardless of induction therapy. Less than $10 \%$ of patients presented bulky and/or multilevel nodes (IIIA4), which were related to a greater risk of incomplete resection,[7] and a significantly higher risk of LRR and death was reported.[25] For that reason, IIIA4 patients could have potential survival benefit from radiotherapy. $\mathrm{Li}$ et al.[26] demonstrated a pathological response rate of $60 \%$ in bulky N2 disease treated by preoperative chemoradiation. Overall, since we analyzed consecutive data instead of performing matching, a tendency of unsuspected or resectable limited nodes in EGFR-mutant disease was 
uncovered. Baba T et al.[27] also noted that positive nodes from adenocarcinoma were likely to be restricted in the low-risk node zone. This evidence might explain the favorable local control in our population, and the reason why only a small portion of patients received neoadjuvant therapy or PORT in clinical practice.

Extranodal extension could be recognized as a predictor of LRR in EGFR-mutant stage III-pN2 lung adenocarcinoma.[28,29] Future studies assessing optimal radiotherapy should consider the stratification of capsule status. Although positive capsule is regarded as a potential indicator of PORT, further validation would be essential, because paradoxical results from another study suggested that PORT could only benefit those without extracapsular invasion.[30] In recent studies, EBUS-TBNA showed an increased capacity in detecting the eventual presence of extracapsular extension,[31,32] making it possible to investigate neoadjuvant radiotherapy in pre-surgically diagnosed extracapsular disease.

A nomogram predicting 1-, 2- and 3-year LRR rates was created, incorporating N2 status and pathological extranodal extension. We then divided patients into two groups with a cut-off value of the 2 -year predicted LRR incidence of $10 \%$. PORT might be spared in those without any of the risk factors (2-year incidence of LRR $\leqslant 10 \%$ ) to avoid radiation-induced injury and improve the quality of life.[33] For those with pre-treatment bulky/multilevel $\mathrm{N} 2$ and/or extranodal extension (2-year incidence of LRR $>10 \%$ ), a propensity-scorematching study will be performed assessing the efficacy and toxicities of PORT. A peer report from Zhang $\mathrm{Y}$ et al. developed nomograms to predict the conditional risk of relapse in completely resected adenocarcinoma as well, including sex, age, tumor size, smoking history, tumor histology, visceral pleural invasion, lymphovascular invasion, and pathologic TNM stage as risk factors. The c-index was 0.743 predicting the overall risk of relapse. However, the study did not create a LRR risk nomogram for future investigation on intensive adjuvant local therapy.[34]

The current study had several limitations. First, $33.7 \%$ of patients, with initial suspected N2 proceeded directly to surgery instead of neoadjuvant treatments after the evaluation by thoracic surgeons.[35,36] However, survival benefit could be found following surgery even in those patients with persistent single N2 involvement after induction therapy,[37,38] leaving primary surgery as a possible choice. A second limitation was that, either ARMS or direct sequencing had been used because both of the techniques were accessible in different cancer centers.
ARMS is believed to identify EGFR mutations more frequently, and those identified by ARMS tend to benefit more from TKIs,[39] which might cause bias on estimating the effect of TKIs on LRR. Finally, as a retrospective study, selection bias and missing data were inevitable. Overall, the results should be validated in an external database and by randomized controlled trials.

\section{Conclusions}

The low incidence of locoregional recurrence after complete resection reflects the distinctive nature of EGFR-mutant III-pN2 lung adenocarcinoma. Intensive adjuvant therapies such as PORT should only be considered in high-risk patients with pre-treatment bulky/multilevel N2 and/or pathological extra-nodal extension determined by the nomogram. Further study evaluating the optimal postoperative approach for completely resected EGFR-mutant N2-positive lung adenocarcinoma is warranted in the high-risk patients.

\section{Abbreviations}

ARMS: amplification-refractory mutation system; CI: confidential interval; c-index: concordance index; CT: computed tomography; DM: distant metastasis; DFS: disease-free survival; EBUS-TBNA: endobronchial ultrasound-guided transbronchial needle aspiration; EGFR: epidermal growth factor receptor; ESMO: European Society for Medical Oncology; HR: hazard ratio; IIIA1-2: unforeseen N2; IIIA3: minimal/single station N2; IIIA4: bulky and/or multilevel N2; KPS: Karnofsky performance score; LRR: locoregional recurrence; MRI: magnetic resonance imaging; OS: overall survival; PET: positron emission tomography; PORT: postoperative radiotherapy; TKI: tyrosine kinase inhibitor.

\section{Supplementary Material}

Supplementary tables.

http://www.jcancer.org/v11p6114s1.pdf

\section{Acknowledgements}

This work was supported by the Science and Technology Planning Project of Guangdong Province, China [grant number 2016A020215190]; the Natural Science Foundation of Guangdong Province [grant number 2018A030313021].

\section{Competing Interests}

The authors have declared that no competing interest exists. 


\section{References}

1. Wang T, Nelson RA, Bogardus A, Grannis FW Jr. Five-year lung cancer survival: which advanced stage nonsmall cell lung cancer patients attain long-term survival? Cancer. 2010;116:1518-25.

2. Feng W, Fu XL, Cai XW, et al. Patterns of local-regional failure in completely resected stage IIIA(N2) non-small cell lung cancer cases: implications for postoperative radiation therapy clinical target volume design. Int J Radiat Oncol Biol Phys. 2014;88:1100-7.

3. Douillard JY, Rosell R, De Lena M, et al. Impact of postoperative radiation therapy on survival in patients with complete resection and stage I, II, or IIIA non-small-cell lung cancer treated with adjuvant chemotherapy: the adjuvant Navelbine International Trialist Association (ANITA) Randomized Trial. Int J Radiat Oncol Biol Phys. 2008;72:695-701.

4. Lally BE, Zelterman D, Colasanto JM, Haffty BG, Detterbeck FC, Wilson LD. Postoperative radiotherapy for stage II or III non-small-cell lung cancer using the surveillance, epidemiology, and end results database. J Clin Oncol. 2006;24:2998-3006.

5. Corso CD, Rutter CE, Wilson LD, Kim AW, Decker RH, Husain ZA. Re-evaluation of the role of postoperative radiotherapy and the impact of radiation dose for non-small-cell lung cancer using the National Cancer Database. J Thorac Oncol. 2015;10:148-55.

6. [Internet] National Comprehensive Cancer Network. Non-Small Cell Lung Cancer version 6. https://www.nccn.org/professionals/physician_gls/ pdf/nscl.pdf

7. Postmus PE, Kerr KM, Oudkerk M, et al. Early and locally advanced non-small-cell lung cancer (NSCLC): ESMO Clinical Practice Guidelines for diagnosis, treatment and follow-up. Ann Oncol. 2017;28(suppl_4):iv1-iv21.

8. Kris MG, Gaspar LE, Chaft JE, et al. Adjuvant Systemic Therapy and Adjuvant Radiation Therapy for Stage I to IIIA Completely Resected Non-Small-Cell Lung Cancers: American Society of Clinical Oncology/Cancer Care Ontario Clinical Practice Guideline Update. J Clin Oncol. 2017;35:2960-74.

9. Yuan C, Tao X, Zheng D, et al. The lymph node status and histologic subtypes influenced the effect of postoperative radiotherapy on patients with N2 positive IIIA non-small cell lung cancer. J Surg Oncol. 2019;119:379-87.

10. Liu SR, Qiu B, Yang H, et al. Epidermal Growth Factor Receptor Mutation Status and Treatment Outcome for R0-Resected Patients with Stage 3 Non-small Cell Lung Cancer. Ann Surg Oncol. 2016;23:2115-22.

11. Zhong WZ, Wang $Q$, Mao WM, et al. Gefitinib versus vinorelbine plus cisplatin as adjuvant treatment for stage II-IIIA (N1-N2) EGFR-mutant NSCLC (ADJUVANT/CTONG1104): a randomised, open-label, phase 3 study. Lancet Oncol. 2018;19:139-48.

12. Yue DS, Xu SD, Wang Q, et al. Erlotinib versus vinorelbine plus cisplatin as adjuvant therapy in Chinese patients with stage IIIA EGFR mutation-positive non-small-cell lung cancer (EVAN): a randomised, open-label, phase 2 trial. Lancet Respir Med. 2018;6:863-73.

13. Amin MB, Edge SB, Greene FL, et al. AJCC cancer staging manual. 8th ed. New York, USA: Springer Verlag; 2017.

14. Robinson LA, Ruckdeschel JC, Wagner H, Stevens CW. Treatment of non-small cell lung cancer-stage IIIA - ACCP evidence-based clinical practice guidelines (2nd edition). Chest. 2007;132(3 Suppl): 243S-265S.

15. Liang W, He J, Shen Y, et al. Impact of Examined Lymph Node Count on Precise Staging and Long-Term Survival of Resected Non-Small-Cell Lung Cancer: A Population Study of the US SEER Database and a Chinese Multi-Institutional Registry. J Clin Oncol. 2017;35:1162-70.

16. Riquet $\mathrm{M}$, Assouad J, Bagan $\mathrm{P}$, et al. Skip mediastinal lymph node metastasis and lung cancer: a particular N2 subgroup with a better prognosis. Ann Thorac Surg. 2005;79:225-33.

17. Yano T, Okamoto T, Fukuyama S, Maehara Y. Therapeutic strategy for postoperative recurrence in patients with non-small cell lung cancer. World J Clin Oncol. 2014;5:1048-54.

18. Pintilie M. Competing risks: a practical perspective. Chichester, England: John Wiley \& Sons; 2006.

19. Huang XD, Zhou GQ Lv JW, et al Competing risk nomograms for nasopharyngeal carcinoma in the intensity-modulated radiotherapy era: A big-data, intelligence platform-based analysis. Radiother Oncol. 2018;129:389-95.

20. Mak RH, Doran E, Muzikansky A, et al. Outcomes after combined modality therapy for EGFR-mutant and wild-type locally advanced NSCLC. Oncologist. 2011;16:886-95.

21. Takamochi K, Oh S, Suzuki K. Prognostic evaluation of nodal staging based on the new IASLC lymph node map for lung cancer. Thorac Cardiovasc Surg. 2010;58:345-9.

22. Detterbeck F. What to do with "Surprise" N2?: intraoperative management of patients with non-small cell lung cancer. J Thorac Oncol. 2008:3:289-302.

23. Kawasaki K, Sato Y, Suzuki Y, Saito H, Nomura Y, Yoshida Y. Prognostic Factors for Surgically Resected N2 Non-small Cell Lung Cancer. Ann Thorac Cardiovasc Surg. 2015;21:217-22.

24. Sun JM, Noh JM, Oh D, et al. Randomized Phase II Trial Comparing Chemoradiotherapy with Chemotherapy for Completely Resected Unsuspected N2-Positive Non-Small Cell Lung Cancer. J Thorac Oncol. 2017;12:1806-13.

25. Decaluwé H, De Leyn P, Vansteenkiste J, et al. Degree of mediastinal lymph node involvement and impact on survival. Surgical multimodality treatment for baseline resectable stage IIIA-N2 non-small cell lung cancer. Eur J Cardiothorac Surg. 2009;36:433-9.

26. $\mathrm{Li} \mathrm{J}, \mathrm{Dai} \mathrm{CH}, \mathrm{Yu} \mathrm{LC}$, et al. Results of trimodality therapy in patients with stage IIIA (N2-bulky) and stage IIIB non-small-cell lung cancer. Clin Lung Cancer. 2009;10:353-9.

27. Baba $T$, Uramoto $H$, Kuwata $T$, et al. Survival impact of node zone classification in resected pathological N2 non-small cell lung cancer. Interact Cardiovasc Thorac Surg. 2012;14:760-4.

28. Luchini C, Veronese N, Nottegar A, et al. Extranodal extension of nodal metastases is a poor prognostic moderator in non-small cell lung cancer: a meta-analysis. Virchows Arch. 2018;472:939-47.

29. Liu W, Shao Y, Guan B, et al. Extracapsular extension is a powerful prognostic factor in stage IIA-IIIA non-small cell lung cancer patients with completely resection. Int J Clin Exp Pathol. 2015;8:11268-77.

30. Moretti L, Yu DS, Chen H, et al. Prognostic factors for resected non-small cell lung cancer with pN2 status: implications for use of postoperative radiotherapy. Oncologist. 2009;14:1106-15.

31. Lilo MT, Allison DB, Younes BK, et al. The critical role of EBUS-TBNA cytology in the staging of mediastinal lymph nodes in lung cancer patients: a correlation study with positron emission tomography findings. Cancer Cytopathol. 2017;125:717-25.

32. Cetinkaya E, Usluer O, Yilmaz A, et al. Is endobronchial ultrasound-guided transbronchial needle aspiration an effective diagnostic procedure in restaging of non-small cell lung cancer patients? Endosc Ultrasound. 2017;6:162-7.

33. Jiang ZQ, Yang $K$, Komaki $R$, et al. Long-term clinical outcome of intensity-modulated radiotherapy for inoperable non-small cell lung cancer: the MD Anderson experience. Int J Radiat Oncol Biol Phys. 2012;83:332-9.

34. Zhang B, Yuan Z, Zhao L, Pang Q, Wang P. Nomograms for predicting progression and efficacy of post-operation radiotherapy in IIIA-pN2 non-small cell lung cancer patients. Oncotarget. 2017;8:37208-16.

35. Van Meerbeeck JP, Kramer GW, Van Schil PE, De Leyn P. Randomized controlled trial of resection versus radiotherapy after induction chemotherapy in stage IIIA-N2 non-small-cell lung cancer. J Natl Cancer Inst. 2007;99:442-50.

36. Albain KS, Swann RS, Rusch VW, et al. Radiotherapy plus chemotherapy with or without surgical resection for stage III non-small-cell lung cancer: a Phase III randomized controlled trial. Lancet. 2009;374:379-86.

37. Cerfolio RJ, Maniscalco L, Bryant AS. The treatment of patients with stage IIIA non-small-cell lung cancer from N2 disease: who returns to the surgical arena and who survives. Ann Thorac Surg. 2008;86:912-20.

38. Decaluwe H, De Leyn P, Vansteenkiste J, et al. Surgical multimodality treatment for baseline resectable stage IIIA-N2 non-small-cell lung cancer. Degree of mediastinal lymph node involvement and impact on survival. Eur J Cardiothorac Surg. 2009;36:433-9.

39. Feng Q, Yang ZY, Zhang JT, Tang JL. Comparison of direct sequencing and amplification refractory mutation system for detecting epidermal growth factor receptor mutation in non-small-cell lung cancer patients: a systematic review and meta-analysis. Oncotarget. 2017;8:59552-62. 